\title{
Nonhyperbolic escape and changes in phase-space stability structures in laser-induced multiphoton dissociation of a diatomic molecule
}

\author{
Vassilios Constantoudis ${ }^{1,2, *}$ and Cleanthes A. Nicolaides ${ }^{1,2, \dagger}$ \\ ${ }^{1}$ Physics Department, National Technical University, Athens, Greece \\ ${ }^{2}$ Theoretical and Physical Chemistry Institute, Hellenic Research Foundation, 48 Vassileos Constantinou Avenue, \\ Athens 11635, Greece
}

(Received 19 April 2001; published 22 October 2001)

\begin{abstract}
The dependence of photodissociation of a diatomic molecule (vibrating according to the Morse potential) on the frequency of the laser field that induces it, is studied in the context of classical nonlinear dynamics. First, it is observed that as the laser frequency increases towards the harmonic frequency of the potential, a transition from stabilization due to Kolmogorov-Arnold-Moser tori to stabilization caused by the resonance stability island occurs. Then, considering the photodissociation as a nonhyperbolic half-scattering process, we investigate the influence of these changes in the phase space stability structures on dissociation dynamics via the examination of the fractal set of singularities appearing in the time-delay function of the initial state. It is found that the effective fractal dimension of this set (a finite-scale approximation of the exact dimension which is always equal to 1) and the percentage of its singularities provide a link between these changes and the dissociation rate.
\end{abstract}

DOI: 10.1103/PhysRevE.64.056211

PACS number(s): 05.45.Df, 33.80.Gj

\section{INTRODUCTION}

During the last fifteen years, the influence of nonlinearity on scattering processes has been studied extensively in the context of classical dynamics [1]. A universal feature of this influence is the appearance of a fractal set of singularities in scattering functions. The scattering functions represent the dependence of some output variable characterizing the trajectory after scattering (e.g., time delay, scattering angle) on some input variable contributing to the initial condition of the trajectory. The trajectory can initially be located either outside the scattering region (scattering processes) or inside it (half-scattering processes). In both cases, the presence of fractal singularities in scattering functions defines chaotic scattering, whereby very small changes in the initial conditions may result in large changes in the output variable.

It has been established that chaotic scattering is due to the existence of nonattracting chaotic invariant sets in phase space containing an infinite number of unstable periodic orbits. The dynamics on this set is classified as either hyperbolic or nonhyperbolic. In the hyperbolic chaotic scattering, there are no tori in the scattering region and all periodic orbits are unstable. The typical survival probability function decays exponentially and the dimension of the fractal set of singularities is less than 1 . On the other hand, nonhyperbolic chaotic scattering is connected to the presence of stable periodic orbits in the scattering region and, as current evidence indicates, is characterized by power law decay of the survival probability due to the stickiness effects of the stable regions surrounding the stable periodic orbits. Moreover, it has been proposed [2] and verified numerically from the study of real or model systems [2-4] that the fractal dimension of the singularities is always 1 , even though their Lebesgue measure remains zero.

\footnotetext{
*Email address: vconst@eie.gr

†Email address: can@eie.gr
}

Although the scattering processes encountered in most physical situations are typically nonhyperbolic, the vast majority of recent research has focused on hyperbolic cases [1]. For example, the evolution of hyperbolic chaotic scattering as a function of a parameter has been studied extensively [5-8], whereas, to the best of our knowledge, the corresponding problem in nonhyperbolic dynamics, as it will be stated below, has not been addressed yet, although some attention has been paid to the more general problem of the transition from hyperbolic to nonhyperbolic scattering $[4,9]$. Since parameter changes in nonhyperbolic dynamics are associated with variations in the stability structures in phase space, the problem actually has to do with the influence of these variations on the scattering dynamics. The study of this problem is motivated not only by the theoretical need to complete the theory of chaotic scattering, but also, and most importantly, by the fact that many scattering systems depend on parameters whose variation modifies the structure of stable regions. Thus, the aim of this paper is to investigate this problem with respect to a real physical process, namely, that of the multiphoton dissociation of a diatomic molecule induced by a strong laser field. In the context of classical dynamics, this process can be considered as a nonhyperbolic half-scattering process, where the laser frequency plays the role of the parameter whose variation modifies the features of the stable regions.

If we focus our attention on a line of initial conditions in phase space representing the initial state, then the influence of the movement and deformation of the stability islands on the escape dynamics is determined by two factors: the relative position of this line with respect to the stable regions in phase space and the strength of the stickiness effects [10] in the borders of nearby stable regions. It will be shown that the role of these factors is revealed by examining the changes in the structure of the set of singularities present in the timedelay functions of the initial state, which serve as a fingerprint of the corresponding changes in the chaotic invariant set in phase space. These changes, however, cannot be quan- 
tified by the exact value of the fractal dimension since, as we said above, it is always equal to 1 for nonhyperbolic systems and hence irrelevant to the changes in the singular set. On the contrary, the effective fractal dimension $d_{\mathrm{eff}}$, which is an approximation to the exact value since it is estimated for a particular range of finite scales, will be shown to be a good descriptor of the aforementioned changes. The same will be found to be true for the percentage of the singular initial conditions $\mathcal{N}_{\text {up }}$ over the escaping part of the initial state. In particular, when a stable region in phase space approaches the initial state and at the same time the associated stickiness effects are getting increasingly pronounced, an increment of the $d_{\text {eff }}$ of the singular set and a proliferation of the singularities (increment of the percentage $\mathcal{N}_{\text {up }}$ ) take place. This is in fact the situation for the classical dissociation of the ground vibrational state of a diatomic molecule when the laser frequency approaches the harmonic frequency of the molecular potential. Moreover, this behavior seems to be the nonhyperbolic analog of the phenomenon of crisis and enhancement of hyperbolic chaotic scattering $[7,8]$.

An important question concerns the observable consequences of the behavior of $d_{\text {eff }}$ and $\mathcal{N}_{\text {up }}$. It will be shown that both quantities are connected with the classical dissociation rate, $R_{\mathrm{cd}}$, of the initial state. In particular, $R_{\mathrm{cd}}$ decreases exponentially sufficiently accurately as $d_{\text {eff }}$ or $\mathcal{N}_{\text {up }}$ increases. Therefore, we conclude that the effective fractal dimension $d_{\text {eff }}$ and the percentage of the singularities $\mathcal{N}_{\text {up }}$ provide a link between the movement and deformation of the stable regions in phase space induced by the change in some parameter and the behavior of $R_{\mathrm{cd}}$ as a function of this parameter.

The paper is organized as follows. We start in Sec. II by presenting the system we study and by investigating the changes in phase space structure as the frequency of the laser field increases. These changes are associated with the behavior of the dissociation probability $P_{\mathrm{cd}}$ and the red shift of the optimum frequency. In Sec. III, we use the tools of chaotic scattering theory and focus our attention on the structure of the fractal set of singularities of the time-delay functions. In particular, we suggest a link between some characteristics of this structure and the dissociation rate. Finally, Sec. IV contains an outlook of the paper and some thoughts about future research.

\section{PHASE SPACE STRUCTURE VS LASER FREQUENCY}

We consider the pure vibrational motion of a diatomic molecule for a fixed electronic configuration and under the assumption that rotational motion is not excited. Then the molecular potential may be described by a Morse potential [11]

$$
V_{M}(r)=D\left(1-e^{-a\left(r-r_{e}\right)}\right),
$$

where $D$ is the dissociation energy, $r_{e}$ is the equilibrium bond distance, and $\alpha^{-1}$ is the range of the potential. HF, $\mathrm{HCl}$, and $\mathrm{NO}$ are some examples of diatomic molecules where our assumptions are well satisfied. The Hamiltonian of such a molecule in an external laser field can be given by

$$
H(r)=H_{0}(r)-\mu(r) F \cos (f t),
$$

where $H_{0}(r)=p^{2} / 2 m+V_{M}(r)$ is the unperturbed Hamiltonian, $F$ is the amplitude of the electric field of the laser, $f$ is its frequency, $m$ is the reduced mass, and $\mu(r)$ is the dipole function.

For the parameters of the Morse potential we use the values corresponding to the molecule HF [12], whereas for the dipole function we use the same analytical form as in Refs. $[13,14]$,

$$
\mu(r)=A r \exp \left(-\xi r^{4}\right)
$$

with $A=0.4541$ and $\xi=0.0064$ (in a.u.). We have confirmed that the results of our study are not sensitive to small variation of these choices.

The classical dynamics of our system can be obtained by the numerical integration of Hamilton's equations

$$
\begin{gathered}
\dot{r}=\frac{p}{m}, \\
\dot{p}=2 D a e^{-a\left(r-r_{e}\right)}\left(1-e^{-a\left(r-r_{e}\right)}\right) \\
+A e^{-\xi r^{4}}\left(4 \xi r^{4}-1\right) F \cos f t .
\end{gathered}
$$

We can also use the action-angle variables of the unperturbed Morse oscillator

$$
\begin{gathered}
J=-\sqrt{\frac{2 \mu D}{\alpha^{2}}}(1-\sqrt{1-E}), \\
\theta=-\operatorname{sgn}(p) \arccos \left[\frac{1-E}{\sqrt{E}} e^{a\left(r-r_{e}\right)}-\frac{1}{\sqrt{E}}\right],
\end{gathered}
$$

where $E=H_{0} / D$ is the dimensionless energy of the unperturbed molecule. The action-angle variables are limited to the bound part of the unperturbed dynamics $(E<1)$ and this is why we prefer to integrate numerically the Hamilton's equations in $(r, p)$ representation and then transform them via Eq. (4) to the $(\theta, J)$ or $(\theta, E)$ representation.

In order to mimic more effectively the quantum photodissociation process, we choose the initial classical state as an ensemble of initial conditions with the energy of the quantum state and angle variables $\theta$ uniformly distributed between $-\pi$ and $\pi[14,15]$. In the rest of the paper, we consider that initially the molecule vibrates in its ground state with energy $E_{0}$ and interacts with a laser field of a constant and strong (nonperturbative) intensity equal to $7.3 \times 10^{-3}$ a.u. In addition, we focus our study on the frequency region in the neighborhood of the harmonic frequency $f_{0}=\left(2 D \alpha^{2} / m\right)^{1 / 2}$ of the Morse potential, since it has been observed that in this region the photodissociation probability is maximized and the quantum and classical results resemble each other $[14,16]$.

To understand the dependence of photodissociation on the frequency $f$ of the laser field, first we have to examine the movement of the stable regions in phase space as well as the deformation of their size caused by the increment of the frequency $f$. Roughly speaking, the overlap of the line of the initial state with these regions in phase space gives the non- 


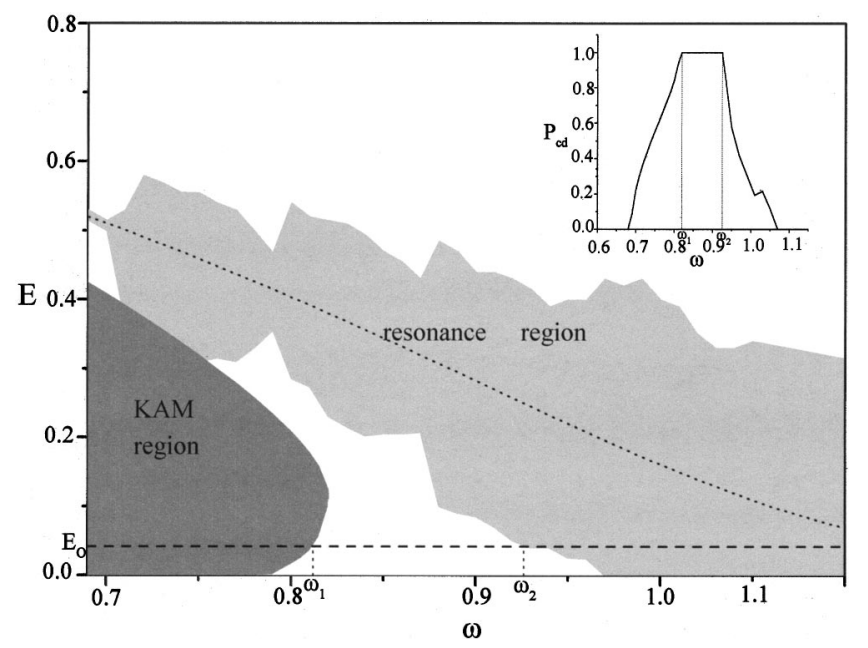

FIG. 1. Energy ranges of KAM and resonance stability regions vs rescaled frequency $\omega=f / f_{0}$. The dashed curve shows the energy $E_{0}$ of the initial state and the dotted curve the energy of the stable periodic orbit of the main resonance. The frequencies $\omega_{1}$ and $\omega_{2}$ mark the transition from KAM to resonant stabilization of the initial state. In between them, no stabilization occurs and the entire initial state dissociates. The inset shows the dependence of the classical photodissociation probability $P_{\mathrm{cd}}$ on the rescaled frequency $\omega$. The $P_{\mathrm{cd}}$ is defined as the percentage of the chaotic and dissociating trajectories over the whole initial state.

dissociating part of the initial state. The remaining part consists of transiently chaotic trajectories, which, after wandering through the interaction region for some time, escape to infinity leading to molecular dissociation. For the particular choice of field intensity and frequency interval, there exist two types of stable regions. The first is the area of KAM tori, which has survived deformed from the unperturbed dynamics, and the second is the stability region surrounding the main resonance $1: 1$ between the field and the internal dynamics. By increasing the frequency towards $f_{0}$, the resonance region is lowered in energy and is enlarged, while the KAM tori area gradually disappears. These changes in the structure of phase space and their effect on the initial state are depicted in Fig. 1, which shows the energy range of the KAM tori (gray region) and the resonant tori (light gray region) for the whole interval of interest of the rescaled frequency $\omega=f / f_{0}$. The dotted curve at the center of the resonance region represents the frequency dependence of the energy of the stable periodic orbit of the resonance, whereas the dashed curve is the line of the initial vibrational state at energy $E_{0}$. By inspecting also the inset in Fig. 1, we can separate the dissociation behavior into three frequency regimes.

In the first regime $\left(\omega<\omega_{1} \cong 0.82\right)$, part of the initial state remains trapped in KAM tori and, consequently, the classical photodissociation probability $P_{\mathrm{cd}}$, the percentage of the transiently chaotic and finally dissociating trajectories, is less than 1. As frequency increases, this part becomes gradually smaller since the KAM area is shrinking more and more, and consequently $P_{\text {cd }}$ increases too. At $\omega=\omega_{1}$ we pass to the second regime. Here the whole classical state can escape to infinity in the limit of infinite time (no overlap takes place), and the $P_{\mathrm{cd}}$ takes on its maximum value $\left(P_{\mathrm{cd}}=1\right)$. This situation holds until $\omega=\omega_{2} \cong 0.925$, where a new overlap phenomenon starts to occur (third frequency regime). The tori of the resonance region, initially lying at higher energies for lower frequencies, now begin to overlap the initial state causing the reduction of the $P_{\mathrm{cd}}$. Finally, at higher frequencies the resonant tori cover the whole classical state and the $P_{\mathrm{cd}}$ vanishes. Not surprisingly, the frequency region, where the probability of photodissociation is maximized, lies slightly to the left of the harmonic frequency $(\omega=1)$. This red shift has been observed in both classical and quantum studies and so cannot be considered an exclusively quantum phenomenon [3,14,17-19]. As we can conclude from inspection of Fig. 1, in classical phase space terms, a transition from KAM to resonant stabilization mechanisms lies behind this phenomenon, and in particular the enlargement of the resonance area as frequency increases.

It is worth noting that the energy range of the resonance area shown in Fig. 1 is actually a rough estimation. The difficulty in locating the outer torus of the resonance region is due to the rich structure occurring at its borders $[20,21]$. This structure involves both cantori (remnants of broken tori) and higher-order resonance zones resulting in strong stickiness effects [10]. On the contrary, for the particular interval of frequency values, the phase space structure around the KAM region is quite different. Stroboscopic plots reveal that no cantori exist and the high-order resonance zones are very narrow. Therefore, the stickiness effects caused by the KAM tori are much weaker than the corresponding effects of the resonance stable region. This difference, combined with the movements of the stable regions described above, has dramatic effects on the dissociation dynamics of the initial state for the frequency region to which we limited our study and sheds light on the dependence of $R_{\mathrm{cd}}$ on the laser frequency. We will explain and quantify these effects as well as their possible relation to the photodissociation rate in the following.

\section{FRACTAL SINGULARITIES AND DISSOCIATION RATE}

In accordance with relevant literature [22,23], we consider that a trajectory escapes from the bound part of the potential leading to dissociation of the molecule when the compensated energy of the molecule becomes greater than $D$. The compensated energy is defined by

$$
E_{C}=\frac{1}{2 m}\left[p-\frac{F}{\omega} A e^{-\xi r^{4}}\left(1-4 \xi r^{4}\right) \sin (\omega t)\right]^{2}+V_{M}(r)
$$

and it is used because it removes the oscillations of the real energy caused by the oscillations of the time-dependent field. Therefore, it helps us to determine the time at which the effect of the molecular potential vanishes. After this time, the molecular bond can be considered broken and hence the elapsed time is in fact the photodissociation time $T_{d}$ of the particular trajectory. Obviously, $R_{\mathrm{cd}}$ depends on the $T_{d}$ 

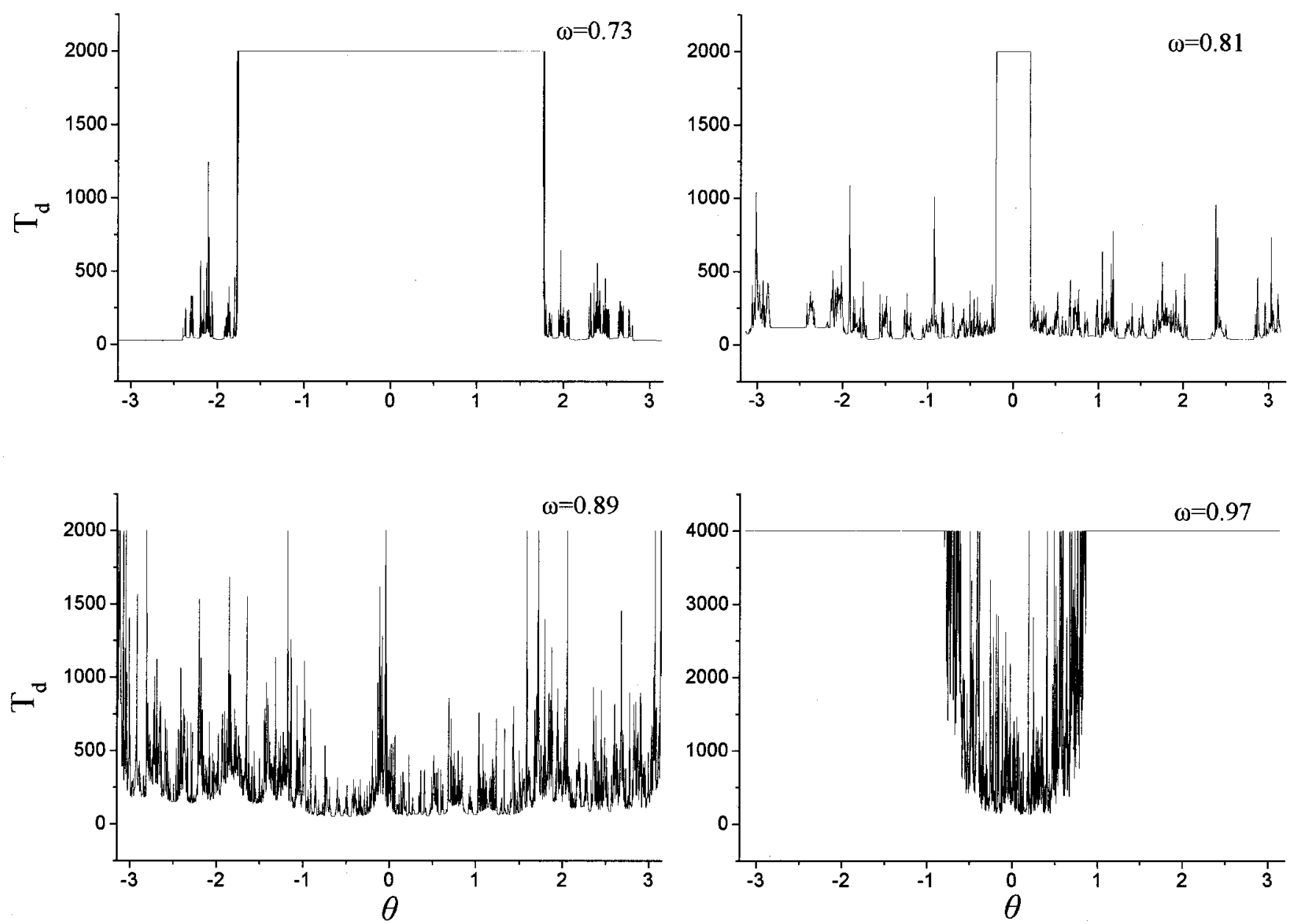

FIG. 2. The time-delay functions $T_{d}(\theta)$ of the initial state with $E=E_{0}$ for increasing values of the rescaled frequency $\omega$. Observe the changes in the magnitude of the dissociating part of the initial state (cf. Fig. 1) and also the modifications in the fractal set of singularities.

of the trajectories that comprise the initial state. Following our previous work [3], we calculate $R_{\mathrm{cd}}$ by applying the form

$$
R_{\mathrm{cd}}=\frac{1}{N} \sum_{i=1}^{N}\left(T_{d}^{(i)}\right)^{-1},
$$

where $T_{d}^{(i)}$ is the photodissociation time of the $i$ th trajectory and the summation is taken over all transiently chaotic and finally dissociating trajectories of the initial state. Remembering that the dissociation process is in fact a half-scattering process, it seems natural to use as a probe the influence of the aforementioned changes in phase space on the features of the time-delay function $T_{d}(\theta)$ of the initial state. This function is characterized by a fractal set of singularities as we can see in Fig. 2, which shows the $T_{d}(\theta)$ for $\omega$ $=0.73,0.81,0.89,0.97$. The transition from KAM to resonant stabilization may also be detected here. At $\omega=0.73$ and 0.81 , the central part of the initial state does not dissociate because of the presence of KAM tori, whereas at $\omega=0.97$ the nondissociating part of the initial state $(|\theta|>1)$ is due to the overlapping with the resonant tori. Apart from this, one can also observe some modifications in the structure of the set of singularities that have to influence the dissociation dynamics. How can we quantify these changes? Usually, the fractality of the set of these singularities is described by the uncertainty dimension $d[24,25]$. In the case of nonhyperbolic dynamics, it has been proposed [2] and numerically verified [2-4] that the exact value of $d$ is always equal to 1 . Obviously, this quantity is irrelevant to our problem since it cannot describe the effect of the movement and deformation of the regular regions on the set of singularities, which determines the behavior of the photodissociation rate. Nevertheless, it is worth noting that $d$ converges to its exact value 1 only when it is computed for successively smaller scales $\varepsilon$, that is, $d \rightarrow 1$ as $\varepsilon \rightarrow 0[2,3]$. For a specific limited range of scales $\varepsilon$, the computed $d$ is actually an approximation from below to the exact value. This approximated or effective uncertainty dimension $d_{\text {eff }}$ is shown to be an important quantity for our problem. We have calculated $d_{\text {eff }}$ for $-12 \leqslant \log _{10}(\varepsilon)$ $\leqslant-4$ as a function of the rescaled laser frequency $\omega$ in the interesting region and the results are shown in Fig. 3 (full circles).

We realize that $d_{\text {eff }}$ is sensitive to the observed changes in the set of singularities caused by the increase in frequency and therefore it can be considered as a good descriptor of the modifications of the chaotic invariant set triggered mainly by the energy descent of the resonant region. Despite the presence of some fluctuations, $d_{\text {eff }}$ increases with increasing frequency. A qualitative explanation of this fact is given below. 


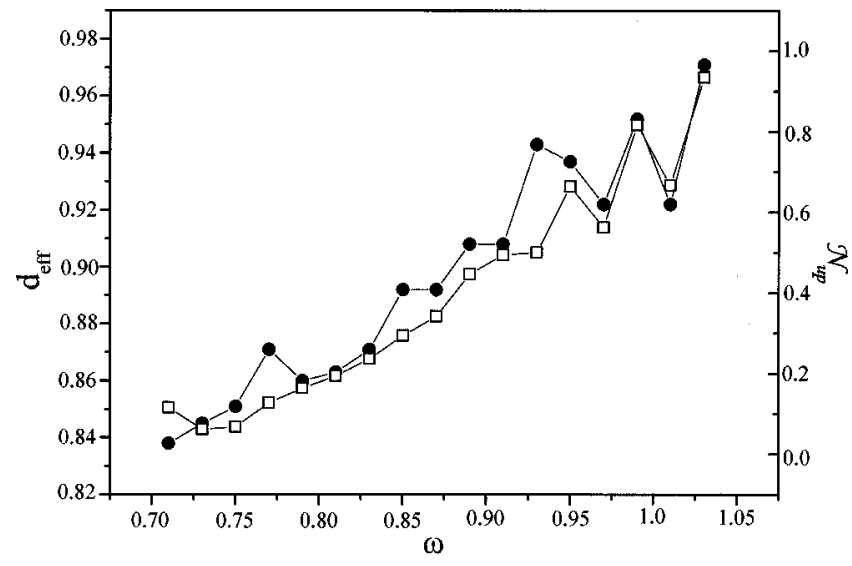

FIG. 3. The effective fractal dimension $d_{\text {eff }}$ (full circles) and the percentage of the singular initial conditions $\mathcal{N}_{\text {up }}$ vs rescaled frequency $\omega$. Notice their sensitivity to $\omega$ despite the nonhyperbolicity of dynamics.

Apart from $d_{\text {eff }}$, the structural changes of stability regions also have an effect on the relative "size" of the fractal set of singularities compared with the dissociating part of the initial state. We can quantify this relative "size" by the percentage of the uncertain points (singular initial conditions) $\mathcal{N}_{\text {up }}(\varepsilon)$ for a particular sufficiently small value of $\varepsilon$. Apparently, $\mathcal{N}_{\text {up }}(\varepsilon)=N_{\text {up }}(\varepsilon) / N_{\text {tc }}$, where $N_{\text {up }}(\varepsilon)$ is the number of the $\varepsilon$-uncertain points and $N_{\text {tc }}$ is the number of the transiently chaotic trajectories. Figure 3 shows the dependence of $\mathcal{N}_{\text {up }}\left(\varepsilon=10^{-6}\right)$ on the rescaled frequency $\omega$ (open squares). We observe that the behavior of the percentage $\mathcal{N}_{\text {up }}(\omega ; \varepsilon)$ is roughly similar to that of $d_{\text {eff }}$. It increases with frequency. Our calculations showed that this behavior is independent of the specific value of $\varepsilon$.

Thus, we conclude that the movement and deformation of the stability regions in phase space influences the structure of the set of singularities in time-delay functions and this effect can be quantified by the effective fractal dimension $d_{\text {eff }}$ and the percentage of the singularities $\mathcal{N}_{\text {up }}$. In particular, because of the weaker stickiness effects around KAM tori, it is mainly the energy descent and the enlargement of the resonant region that makes the fractal set of singularities denser at small scales and more extended in the initial state.

A qualitative explanation of this behavior may be the following. It is well known that the singularities in the scattering functions fall on the intersections of the line of initial conditions with the closure of the stable manifold of the unstable periodic orbits embedded in the nonattracting chaotic invariant set whose existence marks the chaotic scattering. If there were no stable regions in phase space, then the escape dynamics would be hyperbolic and the uncertain dimension would be lower than 1 . The presence of the stability regions is associated with the appearance of stickiness effects [10], according to which some chaotic orbits "stick" to the borders of these regions for some long time interval, leading to a power law behavior of the survival probability. These effects are due to the cantori and the zones of high-order resonances that appear on the borders of stable regions. The manifolds of the unstable periodic orbits of these zones contribute to the formation of the chaotic invariant set providing the nonhyperbolic character of the dynamics. The sticking trajectories actually come from the intersections of these stable manifolds with the line of initial conditions. It is obvious that the influence of the nonhyperbolicity of the dynamics on the initial state depends on the strength of the sticking effects of the stable regions as well as on their relative position with respect to the line of the initial state. It is worth stressing that, the strength of these effects depends not only on the external parameters of the dynamics but also on the type of the stable region. Specifically, in our system and for the particular range of the laser frequency, the stickiness effects around the tori of the main resonance are more pronounced than those of the KAM tori because of the richer structure of cantori and secondary resonances that develop along its borders. Additionally, the enlargement of the stable region is expected to amplify these effects further. Therefore, as regards the nonhyperbolicity of the dynamics, the increment of the frequency causes two effects. First, due to the enlargement of the resonant region, the chaotic invariant set is enriched with the manifolds of the unstable periodic orbits of the high-order resonance zones, and hence its nonhyperbolic character becomes more pronounced. Second, this increasing nonhyperbolicity of the chaotic invariant set becomes more prominent in the initial state as the stable area of the main resonance comes nearer. These effects are quantified by the increment of $d_{\text {eff }}$ and $\mathcal{N}_{\text {up }}$.

The above procedure seems to be, in some sense, the nonhyperbolic analog of the phenomenon of crisis and enhancement of hyperbolic chaotic scattering, which is observed when two isolated chaotic invariant sets become heteroclinically tangent to each other leading to an increment of the uncertainty dimension of the set of singularities as well as to a proliferation of them in the scattering function, the latter called "enhancement of hyperbolic chaotic scattering", $[7,8]$. In the case of the nonhyperbolic dynamics of our system, we roughly have a heteroclinic intersection of the nonhyperbolic invariant set of the unstable periodic orbits of the secondary resonance zones with the hyperbolic invariant set lying in the chaotic region. In accordance with the hyperbolic case, we have found an increment of the effective fractal dimension $d_{\text {eff }}$ and a proliferation of the singularities in the time-delay function expressed by the increment of $\mathcal{N}_{\text {up }}$.

Since the uncertain initial conditions are in fact intersections with the stable manifold of the chaotic invariant set, they will give trajectories acquiring a long dissociation time $T_{d}$. Therefore, a connection between $d_{\text {eff }}$ and $\mathcal{N}_{\text {up }}$, describing the fractal set of singularities, and $R_{\mathrm{cd}}$ is expected to exist. In order to check the validity of this expectation, we calculate $R_{\text {cd }}$ by the formula (3) for the frequency region we used in previous calculations. Figure 4 shows $R_{\mathrm{cd}}$ as a function of $d_{\text {eff }}$ (a) and $\mathcal{N}_{\text {up }}$ (b). We observe that, despite some fluctuations, an exponential relation can clearly be extracted. At present, the exact form is not relevant. The important fact is that the increment of $d_{\text {eff }}$ and $\mathcal{N}_{\text {up }}$ is undoubtedly associated with exponential reduction of $R_{\mathrm{cd}}$. Hence, we can think of the $d_{\text {eff }}$ and $\mathcal{N}_{\text {up }}$ as the quantities connecting the changes in the structure of the stable regions in phase space with the observable photodissociation rate. 

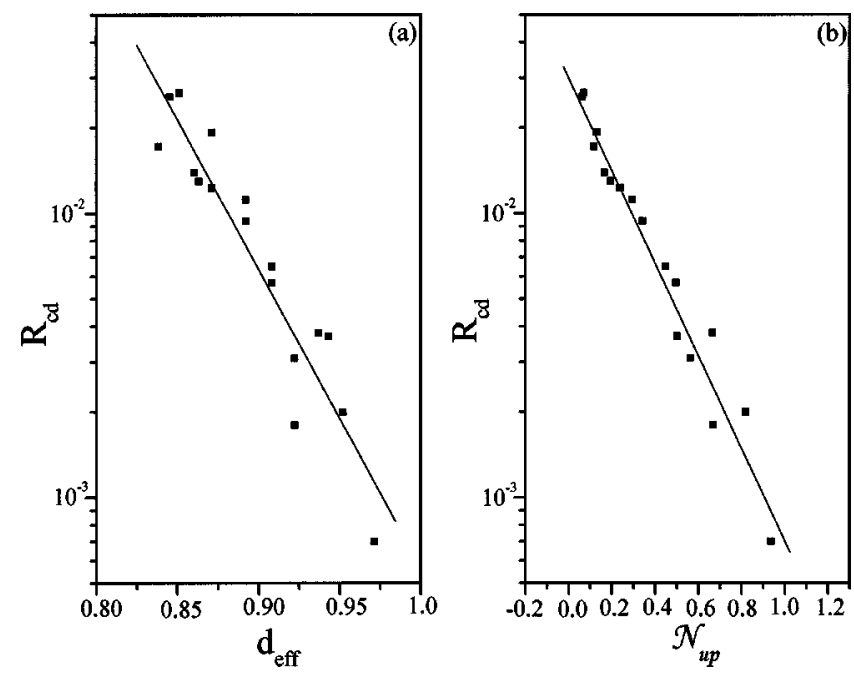

FIG. 4. The exponential dependence of the classical photodissociation rate $R_{\text {cd }}$ on the effective fractal dimension $d_{\text {eff }}$ (a) and on the percentage of the singular initial conditions $\mathcal{N}_{\text {up }}$ (b).

\section{OUTLOOK AND FUTURE PERSPECTIVES}

In this paper, we investigated the classical photodissociation of a Morse diatomic molecule by using the tools of the theory of chaotic scattering. In particular, two questions were addressed. The first referred to the dependence of the phase space stable regions and hence the photodissociation prob- ability on the laser frequency. It was indicated that a transition from KAM to resonance stabilization occurs as the laser frequency $f$ increases towards the harmonic frequency of the potential $f_{0}$, and that at the edge of this transition the photodissociation maximizes. An interesting issue for future study is the quantum mechanical implications of these two different stabilization mechanisms and, in particular, the quantum aspects of the stickiness effects associated with the resonance stability islands.

The second and more important question had to do with the influence of the above phase space changes on the dissociation dynamics and rate. This question was investigated using as a probe the changes in the structure of the fractal set of singularities in the time-delay functions of the initial classical state. We proposed two quantities that can describe these changes, namely, the effective fractal dimension and the percentage of the singular trajectories, and showed that they relate to the classical dissociation rate. The findings concerning the last question may be considered as a first step towards a deeper understanding of the core problem in nonhyperbolic scattering, that is, the influence of the characteristics of the stable regions on scattering dynamics. The second step would be to confirm the generic character of our results by investigating other nonhyperbolic scattering or half-scattering systems. Furthermore, additional analysis involving in a quantitative way the properties of the nonattracting chaotic set and their relation to the dissociation rate would hopefully provide the theoretical background of our results.
[1] P. Gaspard, Chaos, Scattering and Statistical Mechanics (Cambridge University Press, Cambridge, England, 1998).

[2] Y. T. Lau, J. M. Finn, and E. Ott, Phys. Rev. Lett. 66, 978 (1991).

[3] V. Constantoudis and C. A. Nicolaides, Phys. Rev. A 55, 1325 (1997).

[4] S. Drozdz, J. Okolowicz, and T. Srokowski, Phys. Rev. E 48, 4851 (1993).

[5] M. Ding, C. Grebogi, E. Ott, and J. A. Yorke, Phys. Rev. A 42, 7025 (1990).

[6] M. Ding, C. Grebogi, E. Ott, and J. A. Yorke, Phys. Lett. A 153, 21 (1991).

[7] Y. C. Lai, C. Grebogi, R. Blumel, and I. Kan, Phys. Rev. Lett. 71, 2212 (1993).

[8] Y. C. Lai and C. Grebogi, Phys. Rev. E 49, 3761 (1994).

[9] W. Breymann, Z. Kovacs, and T. Tel, Phys. Rev. E 50, 1994 (1994).

[10] C. Efthymiopoulos, G. Contopoulos, N. Voglis, and R. Dvorak, J. Phys. A 30, 8167 (1997).

[11] P. M. Morse, Phys. Rev. 34, 57 (1929); D. ter Haar, Phys. Rev. 70, 222 (1946); H. J. Korch and B. Schellhab, Eur. J. Phys. 21, 73 (2000).

[12] K. P. Huber and G. Herzberg, Molecular Spectra and Molecular Structure. IV. Constants of Diatomic Molecules (Van Nostrant Reinhold, New York, 1979).
[13] J. R. Stine and D. W. Noid, Opt. Commun. 31, 161 (1979).

[14] A. Guldberg and G. D. Billing, Chem. Phys. Lett. 186, 229 (1991).

[15] M. Thachuk and D. M. Wardlaw, J. Chem. Phys. 102, 7462 (1995).

[16] V. Constantoudis and C. A. Nicolaides (unpublished). For the similar problem of the microwave ionization of highly excited hydrogen atoms see P. M. Koch and K. A. H. van Leeuwen, Phys. Rep. 255, 289 (1995).

[17] C. A. Nicolaides, Th. Mercouris, and I. D. Petsalakis, Chem. Phys. Lett. 212, 685 (1993).

[18] P. C. Dardi and S. K. Gray, J. Chem. Phys. 77, 1345 (1982).

[19] R. Heather and H. Metiu, J. Chem. Phys. 88, 5496 (1988).

[20] N. Voglis and C. Efthymiopoulos, J. Phys. A 31, 2913 (1998).

[21] G. Contopoulos, M. Harsoula, N. Voglis, and R. Dvorak, J. Phys. A 32, 5213 (1999).

[22] M. E. Goggin and P. W. Milonni, Phys. Rev. A 37, 796 (1988).

[23] J. G. Leopold and I. C. Percival, Phys. Rev. Lett. 41, 944 (1978).

[24] C. Grebogi, S. W. McDonald, E. Ott, and J. A. Yorke, Phys. Lett. 99A, 415 (1983).

[25] S. Bleher, C. Grebogi, E. Ott, and R. Brown, Phys. Rev. A 38, 930 (1988). 